\title{
DIFERENCIAS POR CULTURA EN UNA VALIDACIÓN INICIAL DE UN INSTRUMENTO EN CONSTRUCCIÓN PARA EVALUAR LA INTELIGENCIA EMOCIONAL EN UNA MUESTRA DE ALUMNOS DE PRIMARIA
}

\author{
Federico Pulido Acosta \\ Departamento de Psicología Evolutiva y de la Educación \\ Universidad de Granada, España \\ (feanor_fede@correo.ugr.es) \\ https://doi.org/10.17060/ijodaep.2017.n1.v3.986
}

Fecha de Recepción: 20 Febrero 2017

Fecha de Admisión: 1 Abril 2017

\section{RESUMEN}

Este trabajo tiene como objetivo elaborar un cuestionario para evaluar la Inteligencia Emocional (IE), siguiendo el modelo que la considera como un conjunto de habilidades, utilizando como referencia el MSCEIT (Mayer Salovey y Caruso, 2009) y destinado a una muestra infantil. Para evitar que la validez del instrumento se pueda ver negativamente afectada se aplicó en análisis a ambos grupos culturales por separado. Para esto se contó con una muestra de 404 participantes, procedentes de 4 centros distintos, de los cuales, el $47.8 \%$ son niños y el $52.2 \%$ niñas y el $68.8 \%$ de cultura/religión musulmana y el 31.2\% cristiana, con edades comprendidas entre los 6 y los 12 años. Como instrumentos de evaluación se emplearon un test elaborado para la evaluación de la IE, junto con una adaptación propia de la EHS (Gismero, 2000). Los resultados obtenidos reflejan niveles de fiabilidad aceptables para ambos grupos culturales. Las dimensiones esperadas fueron diferentes (4 cristianos y 5 musulmanes), apareciendo intercorrelaciones positivas entre los totales y las dimensiones que lo conforman. No fue así para las Habilidades Sociales, medidas a través de este cuestionario. Este cuestionario se muestra como instrumento adecuado para evaluar estas capacidades en la población infantil, tras realizar algunas modificaciones en trabajos futuros, siendo más indicado aplicar el análisis por separado.

Palabras Clave: Emociones, Inteligencia Emocional, educación emocional, instrumento de evaluación, contexto pluricultural.

\section{ABSTRACT \\ Culture differences over initial a validation of a test under construction to asses Emotional Intelligence in Primary degree students.}


The objective of this paper is to develop a questionnaire to assess Emotional Intelligence (El), following the model which sees it as a set of skills, using as reference the MSCEIT (Mayer et al., 2009) aimed at a sample of students from Primary Education. To prevent the validity be adversely affected, we applied analysis to both customs/religion separately. We focused on 404 participants from 6 to 12 years old, from 4 different schools; $42.2 \%$ are boys and $57.8 \%$ girls; $58.9 \%$ are Muslims and $41.1 \%$ Christians. The techniques used in this survey are a test developed to assess El in teenagers and a similar adaptation of EHS (Gismero, 2000). The results show high levels of reliability for both customs/religion groups. The dimensions are different (4 Christians and 5 Muslims) for both groups. There are positive correlations between totals and the dimensions that make them. We found no significant correlations between the two scales. Therefore this questionnaire is an appropriate tool to evaluate these capabilities after making some modifications in future works. It is more appropriate to apply the analysis separately.

Keywords: Emotions, Emotional Intelligence, emotional education, assessment tool, pluricultural context.

\section{INTRODUCCIÓN}

La cambiante actualidad obliga a los Sistemas Educativos a retos cada vez más profundos y audaces, para adaptarse a nuevas formas culturales, sociales y políticas de manera vertiginosa, con el fin último de que el alumnado pueda aprender y desarrollar nuevas habilidades y capacidades que le permita afrontar el futuro con relativa esperanza (Codero y Manchón, 2014).

Actualmente existe un enorme interés por el desarrollo de programas de educación emocional, con independencia de la etapa educativa a la que éste vaya dirigida. Existe, así, una creciente demanda por la implementación de este tipo de programas (Mestre, Guil, Martínez-Cabañas, Larrán \& González, 2011). El desarrollo de todos los componentes emocionales, trabajados en este tipo de experiencias, es un elemento favorecedor de la convivencia entre iguales en la etapa infantil, contribuyendo a la mejora de pautas de pensamiento y comportamientos acordes a las normas sociales, al mismo tiempo que un importante preventor de las conductas violentas dentro del ámbito educativo (Gorostiaga y Balluerka, 2014). Las competencias emocionales son además importantes en el proceso de socialización de los más jóvenes. Todo esto se podrá cumplir si los programas resultan adecuados para las diferentes etapas, por eso los elementos desarrollados deben ser evaluados de manera correcta (Mestre et al., 2011).

Como concepto integrador de todas las capacidades socioemocionales aparece la Inteligencia Emocional, entendida como la habilidad para manejar el propio repertorio emocional, pasando el éxito del ámbito cognitivo al ámbito emocional. Resulta evidente que la manera de entenderla se centra en ésta en cuanto a un conjunto de habilidades que permiten razonar sobre las emociones y emplearlas para la mejora de los procesos cognitivos. Serían aptitudes reales para resolver problemas emocionales (Mayer et al., 2009). A la vez, esta concepción permite que se pueda hablar de un conjunto de habilidades específicas que integran la IE (Pulido y Herrera, 2015), englobando las habilidades de conocimiento de sí mismos, autocontrol y motivación. Estas tres capacidades integran la inteligencia intrapersonal, mientras que la empatía y habilidades sociales integran la intrapersonal.

Teniendo en cuenta lo comentado, se pretende encontrar un instrumento válido para llevar a cabo la evaluación de las mencionadas capacidades, con intención de hacer más adecuados los programas de educación emocional. Este tipo de cuestionarios se centran en la evaluación de este conjunto de aptitudes en una población adulta, existiendo un vacío en la medida de las mismas en poblaciones infantiles y juveniles, las cuales constituyen un importante blanco de este tipo de programas educativos centrados en las emociones. 
Otro de los aspectos más relevantes en relación con la IE es la existencia de diferencias en función del grupo cultural. Desde este punto de vista, se parte de trabajos como el de Pulido y Herrera (2015), Io que no hace otra cosa que alimentar las diferencias entre grupos culturales. Desde esta perspectiva Matesanz (2006), encuentra una importante objeción a los estudios que suelen aplicar el análisis estadístico a muestras, no por separado para ambos grupos culturales. Esto último es teóricamente aconsejable en este tipo de estudios.

Contemplando la enorme incidencia que tiene el entorno cultural sobre las capacidades emocionales, se hace necesario utilizar instrumentos validados transculturalmente, con poblaciones de distintos contextos lingüísticos y culturales, tal y como se recoge en Tobías-Imbernón, OlivaresOlivares y Olivares (2013). De esta manera, se pretende determinar con más precisión el valor predictivo y/o discriminativo dentro de esta dimensión, como reveladora de un rasgo propio de cada grupo cultural. Por esta razón, el presenta trabajo hace especial hincapié en el análisis de los datos con independencia para ambos grupos culturales.

Considerando todo lo mencionado, se presenta el estudio llevado a cabo con una muestra infantil, de alumnos escolarizados en Centros de Educación Infantil y Primaria en la ciudad de Ceuta. Los objetivos del trabajo han sido elaborar y validar un instrumento para evaluar la Inteligencia Emocional considerada como conjunto de habilidades para una población infantil, considerando por separado el análisis de las propiedades psicométricas de ambos grupos culturales.

\section{MÉTODO}

\section{Participantes}

Para afrontar este estudio se seleccionó una muestra integrada por 404 participantes (126 cristianos y 278 musulmanes) que reflejan las características de nuestro contexto pluricultural, repartidos entre los cuatro centros que participaron de manera voluntaria. Las edades de la muestra estaban comprendidas entre los 6 y los 12 años ( $M=9.4$, D.T.=1.63, Rango=6-12). Describiendo la muestra, en función de la variable género, se dan porcentajes bastante equilibrados entre sí, siendo el $47.8 \%$ niños y el resto de la muestra $(52.2 \%)$ chicas. Considerando la cultura el grupo de musulmanes constituye el $68.8 \%$ de la muestra, mientras que el $31.2 \%$ de la misma pertenecía a la cultura-religión cristiana. Los participantes se seleccionaron por el método de muestreo no probabilístico por conveniencia, incidental o casual. Se seleccionó, aleatoriamente, un curso (desde primero a sexto de Primaria) por cada uno de los centros. El error muestral fue del $3 \%$.

Tabla 1.

Muestra en función de variables sociodemográficas

\begin{tabular}{|c|c|c|c|c|}
\hline \multirow[t]{2}{*}{ Variables sociodemográficas } & \multicolumn{4}{|c|}{ Media descriptiva } \\
\hline & & Media & D.T. & Rango \\
\hline \multirow[t]{2}{*}{ Edad } & $\mathrm{N}=404$ & 9.4 & 1.63 & $6-12$ \\
\hline & & & $\mathrm{N}$ & $\%$ \\
\hline \multirow[t]{2}{*}{ Cultura/Religión } & Cristianos & & 126 & 31.2 \\
\hline & Musulmanes & & 278 & 68.8 \\
\hline \multirow[t]{3}{*}{ Sexo } & Niños & & 193 & 47.8 \\
\hline & Niñas & & 211 & 52.2 \\
\hline & Bajo & & 145 & 35.5 \\
\hline \multirow[t]{3}{*}{ Estatus } & Medio-Bajo & & 79 & 19.6 \\
\hline & Medio & & 81 & 20 \\
\hline & Alto & & 99 & 24.5 \\
\hline
\end{tabular}




\section{Instrumento}

La intención fue la de crear un instrumento que permitiera evaluar la Inteligencia Emocional como conjunto de habilidades específicas. Por tanto, este cuestionario pide al sujeto que resuelva problemas emocionales organizados en diferentes secciones. Para ello se tomó como referencia y punto de partida el MSCEIT (Test de Inteligencia Emocional de Mayer et al., 2009), adaptándolo a la población infantil. El instrumento de referencia da una visión integradora de la Inteligencia Emocional, presentándola como un grupo de aptitudes. El problema es que no es apto para su uso en la población infantil. Al mismo tiempo, se pretende incluir entre las capacidades evaluadas (de acuerdo con la concepción de IE defendida en el apartado teórico) las competencias sociales, no consideradas en el cuestionario de referencia.

Para llevar a cabo la elaboración del instrumento se contó con la opinión de dos expertos en los campos de la psicología, las emociones, la educación, la evaluación y la estadística. Partiendo del modelo presente en el MSCEIT (dividido en dos áreas, cuatro ramas y ocho tareas) se siguió la misma estructura del cuestionario original, respetando el número de secciones del mismo (exceptuando la G, que se suprimió por su excesiva dificultad para el alumnado de E. Primaria). En ellas aparecen apartados para la identificación de emociones en gestos faciales y en diferentes fotografías (secciones A y E del cuestionario original), la comprobación de la utilidad de diferentes emociones en situaciones determinadas (sección B), la selección de la emoción que más se ajuste a cada caso (sección C), la respuesta a cuestiones emocionales acordes a determinadas historias (sección $\mathrm{D}$ y H) y la relación de diferentes emociones con acciones concretas (sección F); ajustando las diferentes secciones al nivel del alumnado. Estas corresponden a cada una de las 8 diferentes tareas que aparecen en el cuestionario original. En este instrumento, la sección eliminada (G) fue sustituida por un apartado para evaluar las competencias sociales del sujeto, aprovechando la idea de incluir ítems que evaluasen estas capacidades, no considerados en el cuestionario original.

Con respecto a las diferentes dimensiones, aparecen factores relacionados con la Percepción emocional (Rama 1), al que se vinculó con el Conocimiento de sí mismos. Otra de las dimensiones del cuestionario original se refiere a la Facilitación emocional (Rama 2). En nuestro cuestionario se agruparon por el factor Motivación. La Rama 3 del cuestionario original (Comprensión emocional) quedó dividida, en este caso, en dos factores. Así para la comprensión de información emocional se diferenció entre las propias (Autoconcepto) y las ajenas (Empatía). La 4⿳亠丷a y última Rama del cuestionario de referencia se relaciona con el Manejo emocional. En nuestro caso se empleó el término Autocontrol. El número total de ítems en el cuestionario fue de 58, distribuido entre los diferentes factores. Para el sistema de respuestas es a través de una escala tipo Likert (secciones A,C, F y G) de cuatro puntos o bien a través de la selección de la emoción que más se adapta a cada situación imagen o persona (B, D y E) de un listado de 4 diferentes.

Para las Habilidades Sociales se empleó una adaptación de la EHS (Escala de Habilidades Sociales, Gismero, 2000). Este instrumento quedó integrado por 32 ítems.

\section{Procedimiento}

Una vez obtenidos los datos y construida la base, se llevó a cabo el pertinente análisis estadístico, empleando para ello el Statistical Package for Social Sciences (SPSS 20, 2011). Antes de iniciar el análisis del cuestionario se procedió a realizar una primera valoración para contemplar la existencia de diferencias significativas en los totales de IE empleando como variable independiente la Cultura. Para ello se empleó la prueba ANOVA. Una vez evaluadas estas diferencias se continuó con el análisis de la consistencia interna del cuestionario. Para evaluar la fiabilidad se emplearon la prueba de Cronbach junto con la prueba de dos mitades de Spearman-Brown. Posteriormente se apli- 
caron cálculos para comprobar la validez factorial, empleando un Análisis Factorial Confirmatorio (AFC), por medio del análisis de varianza de componentes principales con rotación varimax. La fiabilidad para cada una de las muestras se comprobó tanto para el cuestionario, como para cada uno de los factores (4-5) obtenidos en cada caso. Para asegurar la validez del modelo jerárquico propuesto, se aplicaron cálculos correlacionales entre el cuestionario (IE) y cada uno de los factores del mismo (incluyendo las Habilidades Sociales). Finalmente se volvió a usar la prueba ANOVA para contemplar la existencia de diferencias significativas en los factores que coinciden en ambas culturas.

\section{RESULTADOS}

Se comienza con los resultados que hacen referencia a la existencia de diferencias significativas para ambos grupos culturales. Los resultados de la prueba ANOVA resaltan la existencia de diferencias significativas en los niveles totales de IE $(p=.000)$, en función de la variable Cultura/Religión. Los musulmanes reflejan un nivel más bajo que los cristianos, tal y como demuestran sus medias (92.89 los musulmanes y 103.26 los cristianos).

Se comienza con los resultados que hacen referencia a la consistencia interna del instrumento. La fiabilidad de la medida de la Inteligencia Emocional, a través del cuestionario elaborado, en su versión inicial, se evaluó, en primer caso a través de la prueba de Cronbach. La consistencia interna del cuestionario (IE) fue de .867 para los cristianos y .875 para la muestra musulmana. En un segundo análisis se procedió a realizar la prueba de dos mitades de Spearman-Brown. Este coeficiente fue de .838 para el cuestionario, entre los musulmanes y .821 entre los cristianos. Se observa una gran igualdad para ambos grupos culturales, incluyendo todos los ítems.

Los factores encontrados entre los cristianos fueron 4. Para el primer factor, la Empatía, su valor fue de .838. La del segundo factor, que aúna elementos relacionados con el Autoconcepto y la Motivación, fue de .755. El tercer factor, que hace referencia al Autocontrol obtuvo una fiabilidad de .731. Para el factor Conocimiento de sí mismos fue de .661, el valor más bajo. Para las Habilidades Sociales, la consistencia interna viene especificada por un de Cronbach de .744. Los resultados del segundo análisis (Spearman-Brown) para los factores fue de .837, .731, .713 y .630 respectivamente (siguiendo el orden en el que se han comentado anteriormente). Para las HHSS fue de .679. Los valores de la primera prueba ( de Cronbach) quedan recogidos en la tabla 2.

Tabla 2.

Valores de la prueba de Cronbach en cuestionario y factores en cristianos

CONSISTENCIA INTERNA DEL CUESTIONARIO

\begin{tabular}{lcc}
\hline Inteligencia Emocional & $\alpha$ de Cronbach & .867 \\
\hline \multicolumn{1}{c}{ CONSISTENCIA INTERNA DE LOS FACTORES } \\
\hline Empatía & $\alpha$ de Cronbach & .838 \\
Motivación/Autoconcepto & $\alpha$ de Cronbach & .755 \\
Autocontrol & $\alpha$ de Cronbach & .731 \\
Conocimiento & $\alpha$ de Cronbach & .661 \\
\hline \multicolumn{1}{c}{ CONSISTENCIA INTERNA HABILIDADES SOCIALES } \\
\hline Habilidades Sociales & $\alpha$ de Cronbach & .744 \\
\hline
\end{tabular}


Los factores en los musulmanes fueron 5. El primero, Motivación, obtuvo un valor de .827, la Empatía, segundo, obtuvo .800. El tercer factor, que hace referencia al Autocontrol obtuvo una fiabilidad de .754, mientras que el cuarto, Autoconcepto, fue de .744. El valor más bajo se obtuvo en Conocimiento de sí mismos (.665). Para las Habilidades Sociales fue de .746. Los coeficientes del segundo análisis para los factores fue de $.843, .761, .725, .733$ y .639 respectivamente. Para las HHSS fue de .698 .

Tabla 3.

Valores de la prueba de Cronbach en cuestionario y factores en musulmanes

\begin{tabular}{lcc}
\hline \multicolumn{3}{c}{ CONSISTENCIA INTERNA DEL CUESTIONARIO } \\
\hline Inteligencia Emocional & $\alpha$ de Cronbach & .875 \\
\hline \multicolumn{1}{c}{ CONSISTENCIA INTERNA DE LOS FACTORES } \\
\hline Motivación & $\alpha$ de Cronbach & .827 \\
Empatía & $\alpha$ de Cronbach & .800 \\
Autocontrol & $\alpha$ de Cronbach & .754 \\
Autoconcepto & $\alpha$ de Cronbach & .744 \\
Conocimiento de sí mismos & $\alpha$ de Cronbach & .665 \\
\hline \multicolumn{1}{c}{ CONSISTENCIA INTERNA HABILIDADES SOCIALES } \\
\hline Habilidades Sociales & $\alpha$ de Cronbach & .746 \\
\hline
\end{tabular}

En el apartado relacionado con la varianza factorial se emplearon diferentes Análisis Factoriales Confirmatorios (AFC). En este sentido, los factores obtenidos entre los cristianos fueron 4. El primero de los cuatro factores está relacionado con el la Empatía, siendo la categoría con mayor número de ítems (18). Entre todos los reactivos ofrecen una varianza explicada del $16.982 \%$. El segundo factor, queda vinculado con el Autoconcepto y la Motivación. Son 15 los ítems que aparecen, que llegan a representar el $10.614 \%$ de la varianza explicada. El Autocontrol, también con 15 ítems. Estos ítems representan el $9.060 \%$ de la varianza explicada. Finalmente aparece el Conocimiento de sí mismos, con el menor número de ítems (10), representando el $6.56 \%$ de la varianza explicada. Entre todos los factores suman una varianza explicada total del $43.216 \%$. Todos los resultados en este apartado aparecen resumidos en la tabla 4.

Tabla 4.

Varianza e ítems de la agrupación por factores del cuestionario en cristianos

\begin{tabular}{lccc}
\hline \multicolumn{4}{c}{ VARIANZA DE FACTORES DE CUESTIONARIO IE } \\
\hline \multicolumn{1}{c}{ FACTOR } & $\mathrm{N}^{\circ}$ ítems & V. Explicada & Suma Total \\
\hline EMPATÍA & 18 & $16.982 \%$ & 58 ítems \\
AUTOCONCEPTO/ MOTIVACIÓN & 15 & $10.614 \%$ & $43.216 \%$ \\
AUTOCONTROL & 15 & $9.060 \%$ & varianza \\
CONOCIMIENTO DE SÍ MISMOS & 10 & $6.560 \%$ & total \\
& & & explicada \\
\hline
\end{tabular}


En este apartado, los factores obtenidos en los musulmanes fueron 5. El primero de los cinco factores, relacionado con la Motivación incluye 13 ítems, que representan el 17.084\% de la varianza explicada. Esta categoría no se puede equiparar con la descrita entre los cristianos, dado que en esta muestra también incorpora ítems relacionados con el Autoconcepto. El segundo de los factores está conformado por 12 ítems (representan el 10.638\% de la varianza explicada), que hacen referencia a la Empatía. Esta categoría incorpora, prácticamente, los mismos ítems en ambas muestras, por lo que es posible vincularlos mutuamente. Exactamente lo mismo ocurre con el tercer factor, que se relaciona el Autocontrol. Son 13 los ítems que lo conforman y representa el $8.457 \%$ de la varianza explicada. El cuarto factor, queda constituido por 8 ítems (es la categoría conformada por el menor número de ítems), sin embargo, no se puede relacionar con el descrito en la muestra cristiana, dado que integra sólo ítems relacionados con el Autoconcepto. Entre estos 8 ítems, vinculados sólo con esta capacidad, se obtiene el $4.572 \%$ de la varianza explicada. El factor final, vinculado al Conocimiento de uno mismo, está formado por 12 ítems, que representan el $3.808 \%$ de la varianza explicada. La mayoría de los ítems (10) coinciden, por lo que ambas categorías se pueden equiparar. Todo esto da una varianza explicada total del $44.599 \%$. Todos los resultados en este apartado aparecen resumidos en la tabla 5.

Tabla 5 .

Varianza e ítems de la agrupación por factores del cuestionario en musulmanes

\begin{tabular}{lccc}
\hline \multicolumn{4}{c}{ VARIANZA DE FACTORES DE CUESTIONARIO IE } \\
\hline \multicolumn{1}{c}{ FACTOR } & $\mathrm{N}^{\mathrm{o}}$ ítems & V. Explicada & Suma Total \\
\hline MOTIVACIÓN & 13 & $17.084 \%$ & 58 ítems \\
EMPATÍA & 12 & $10.638 \%$ & $44.559 \%$ en \\
A. & 13 & $8.457 \%$ & la varianza \\
AUTOCONTROL & 8 & $4.572 \%$ & total \\
AUTOCONCEPTO & 12 & $3.808 \%$ & explicada \\
CONOCIMIENTO DE SÍ MISMOS & & &
\end{tabular}

De la misma manera que hace el MSCEIT, el cuestionario elaborado pretende medir un área unitaria, la Inteligencia Emocional, que a su vez se subdivide en una serie de habilidades específicas. Para que el planteamiento jerárquico propuesto sea válido, es necesario que las diferentes puntuaciones correlacionen positivamente entre sí. La correlación fue significativa al nivel $p=.01$, tal y como era de esperar. Las correlaciones más bajas (en el caso de los cristianos) se dan entre la Inteligencia Emocional y el factor Conocimiento de sí mismos (.493). Fuera de ellas, los resultados se mueven entre el máximo (.689), encontrado en el factor Motivación/Autoconcepto y el mínimo (.622) obtenido entre la Inteligencia Emocional y la Empatía. Los mismos resultados comentados para la Inteligencia Emocional y todos los factores se repiten considerando las intercorrelaciones de los factores entre sí. En todos los casos el nivel de significación es de al menos $p=.01$. En este sentido, los niveles son más bajos que los reflejado para los totales (algo esperado), moviéndose entre los más altos (.500) encontrados en las correlaciones entre Autocontrol y Conocimiento de sí mismos y los más bajos (.290) encontrados entre este último factor y la Empatía. También se realizaron correlaciones considerando las Habilidades Sociales, sin embargo estas (-.089) indican la ausencia de relación entre la IE y las Habilidades Sociales, empleando el cuestionario comentado. 
Tabla 6.

Correlaciones factores IE cristianos

\begin{tabular}{lccccc}
\hline & IE & Empa. & Mo/Au Aucotr.Conoci. \\
\hline Inteligencia Emocional & 1 & & & & \\
Empatía & .622 & 1 & & & \\
Motivación/Autoconcepto & .689 & .345 & 1 & & \\
Autocontrol & .638 & .323 & .356 & 1 & \\
Conocimiento de sí mismos & .493 & .290 & .315 & .500 & 1 \\
La correlación es significante al nivel & 0.01. & & & \\
\hline
\end{tabular}

De la misma manera, se valoraron estos resultados en la muestra musulmana. La correlación volvió a ser significativa al nivel $p=.01$. Las correlaciones más altas se dan entre la Inteligencia Emocional y el factor Motivación (.737). Los resultados más bajos (.488) se obtienen entre la Inteligencia Emocional y el Autoconcepto. Lo mismo ocurre en todos los factores ( $p=.01)$. En este sentido, se mueven entre los más altos (.434) encontrados en las correlaciones entre la Motivación y el Autocontrol y los más bajos (.196) encontrados entre el Autoconcepto y el Autocontrol. Lo mismo comentado en los cristianos se obtuvo en esta muestra, en el caso de las Habilidades Sociales (-.049). Los resultados comentados aparecen resumidos en la tabla 7.

Tabla 7

Correlaciones factores IE musulmanes

\begin{tabular}{lcccccc}
\hline & \multicolumn{1}{c}{ IE } & Motiv. & Empa. Autrol. Aucep. Conoc. \\
\hline Inteligencia Emocional & 1 & & & & & \\
Motivación & .737 & 1 & & & & \\
Empatía & .571 & .322 & 1 & & & \\
Autocontrol & .585 & .434 & .220 & 1 & & \\
Autoconcepto & .488 & .271 & .279 & .196 & 1 & \\
Conocimiento de sí mismos & .621 & .368 & .343 & .281 & .240 & 1 \\
La correlación es significante al nivel 0.01. & & & & \\
\hline
\end{tabular}

A continuación se comentan los resultados de la prueba ANOVA, con la intención de comprobar la existencia de diferencias significativas en función de la variable Cultura/Religión. En este sentido, se consideraron sólo los factores que coinciden en ambos grupos culturales (con los mismos ítems). Aparecen tres categorías. En todas ellas (Autocontrol $-p=.000-$, Empatía $-p=.002-\mathrm{y}$ Conocimiento de sí mismos - $p=.006-)$ se obtienen diferencias significativas, teniendo los musulmanes niveles más bajos que los cristianos, tal y como indican sus medias (los musulmanes .80 y los cristianos .94 en Autocontrol, 2.38 cristianos y 2.12 musulmanes en Empatía y 1.76 los cristianos y 1.30 los musulmanes en Conocimiento de sí mismos). 


\section{CONCLUSIONES}

Partimos en el presente estudio con la idea de hacer una validación inicial de un instrumento para evaluar la Inteligencia Emocional, en una población infantil. Con la intención de aumentar su validez y poder predictivo se analizaron por separado las muestras de culturas diferentes. Los niveles de consistencia interna del instrumento fueron aceptables para la Inteligencia Emocional. En ambos grupos culturales son muy similares ( $\alpha=.867$ los cristianos y $\alpha=.875$ los musulmanes). Respecto a la dimensionalidad del instrumento, se encontraron pocas diferencias entre ambos grupos culturales. En el caso de los musulmanes se obtuvieron 5 factores. Estos factores son Motivación (el factor de los cristianos incluye el Autoconcepto), Empatía (primera categoría en los cristianos), Autocontrol (también tercera categoría en cristianos), Autoconcepto (tampoco se pueden equiparar, dado que los ítems incluyen Motivación en los cristianos) y Conocimiento de sí mismos (último factor de los cristianos). Entre todos los factores suman una varianza explicada total del $44.599 \%$ para los musulmanes y de 43.216 para cristianos, quedando el cuestionario integrado por un total de 58 ítems, en ambos casos. Estos resultados, unidos a los referentes a las correlaciones (significativos al nivel $p=.01$ en todos los casos) que se dan entre la Inteligencia Emocional y cada uno de los factores, de la misma manera que los obtenidos para las intercorrelaciones entre factores, apoyan el concepto jerárquico propuesto. Se encontraron diferencias significativas por Cultura en los totales y todas las categorías que coincidían en ambas muestras (3), actuando todas en una misma dirección: en todos los casos los cristianos obtienen mejores resultados. Diferencias en las habilidades emocionales entre dos grupos culturales también se encontraron en Pulido y Herrera (2015), donde se señalan las dificultades para la integración socio educativa y las diferencias de estatus socioeconómico y cultural (Hernández, Rodríguez y Moral, 2011) como importantes justificantes de estas diferencias, junto con la diversidad encontrada en los núcleos familiares, con estilos de crianza distintos y las importantes diferencias en la lengua materna (Pulido y Herrera, 2015) entre ambas etnias, contribuyen a la aparición de problemas de integración socioeducativa del alumnado no autóctono. Ante esta situación, la interculturalidad se antoja necesaria aunque no suficiente para la progresiva eliminación de estas diferencias, convirtiendo ese contexto en "autóctono" para todos los grupos culturales. El contexto "autóctono" se mostraría como un impulso para el desarrollo socioafectivo (Pulido y Herrera, 2015).

Finalmente indicar que, para ser la primera validación, los resultados iniciales nos permiten ser optimistas con respecto a los elementos a corregir para depurar este instrumento de evaluación de las habilidades emocionales. Del mismo modo, puede ser un importante cuestionario a emplear dentro del ámbito psicológico, social y, sobre todo, educativo en una población infantojuvenil y multicultural, superando elementos como la complejidad para leer y entender los ítems en el cuestionario de referencia, destinado a una población adulta. Con respecto a las diferencias entre musulmanes y cristianos, estas aparecen (sólo varían 2 factores), por lo que, siguiendo a Matesanz (2006) se destaca una mayor precisión y más valor predictivo, siendo la Cultura un elemento discriminativo revelador de un rasgo propio de cada grupo cultural. Para evitar que la validez del instrumento se pueda ver negativamente afectada, lo más indicado es aplicar el análisis estadístico por separado para ambos grupos culturales.

\section{REFERENCIAS BIBLIOGRÁFICAS}

Billings, C., Downey, L. A., Lomas, J. E., Lloyd, J. y Stough, C. (2014). Emotional Intelligence and scholastic achievement in pre-adolescent children. Personality and Individual Differences, 65, 14-18.

Brouzos, A., Misalidi, P. y Hadjimatheou, A. (2014). Associations Between Emotional Intelligence, 
Socio-Emotional Adjustment, and Academic Achievement in Childhood: The Influence of Age. Canadian Journal of School Psychology, 29(2), 83-99.

Codero, J. M. y Manchón, C. (2014). Factores explicativos del rendimiento en educación primaria: un análisis a partir de TIMSS 2011. Estudios Sobre Educación, 27, 9-35

Gismero E. (2000). EHS, Escala de Habilidades Sociales. Madrid: TEA Publicaciones de Psicología Aplicada.

Gorostiaga, A. y Balluerka, N. (2014). Evaluación de la empatía en el ámbito educativo y su relación con la inteligencia emocional. Revista de Educación, 364, 12-38.

Hernández, E., Rodríguez, F. J. y Moral, M. V. (2011). Adaptación escolar de la etnia gitana: relevancia de las variables psicosociales determinantes. Apuntes de Psicología, 29(1), 87-105.

Matesanz, A. (2006). Datos para la adaptación castellana de la Escala de Temores (FSS). Análisis y Modificación de Conducta, 32, 144, 521-551.

Mayer, J., Salovey, P. y Caruso, D. (2009). Test de Inteligencia Emocional de Mayer, Salovey y Caruso. Madrid: TEA Ediciones.

Mestre, J. M., Guil, R., Martínez-Cabañas, F., Larrán, C. \& González, G. (2011). Validación de una prueba para evaluar la capacidad de percibir, expresar y valorar emociones en niños de la etapa infantil. Revista Electrónica Interuniversitaria de Formación del Profesorado, 14(3), 37-54. Recuperado de web: http://www.aufop.com

Pérez, N. y Castejón, J. L. (2006). Relaciones entre la Inteligencia Emocional y el Cociente Intelectual con el rendimiento académico en estudiantes universitarios. Revista Electrónica de Motivación y Emoción (R.E.M.E.), 9(22) 1-27.

Pulido, F. y Herrera, F. (2015). Miedo e inteligencia emocional en el contexto pluricultural de Ceuta. Anuario de Psicología, 45(2), 249-263.

SPSS Inc. Released 2011. IBM SPSS Statistics for Windows, Version 20.0. Armonk, NY: IBM Corp. Tobías-Imbernón, C., Olivares-Olivares, P. J. y Olivares, J. (2013). Revisión de autoinformes de fobia social diseñados para niños y adolescentes. Revista Mexicana de Psicología, 30(2), 123-130. 